\title{
Peptic ulcer associated with COVID-19 in Saudi Arabia
}

Ghada A. Merdad, MD, MSc, Lamees E. Seadawi, MD, Abdulmoneim A. Mustafa, MD.

\begin{abstract}

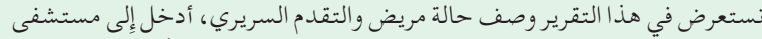

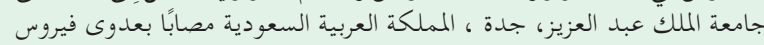

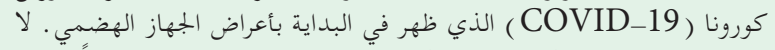

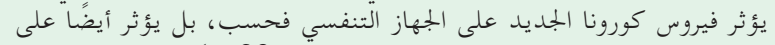

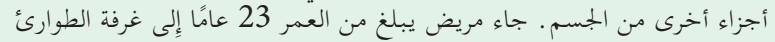

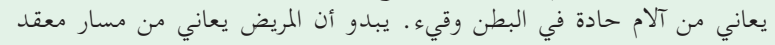

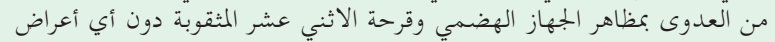

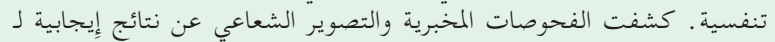

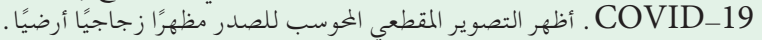

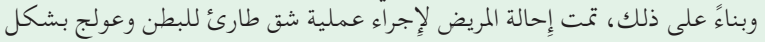

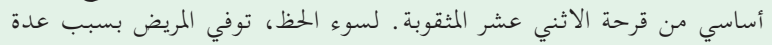

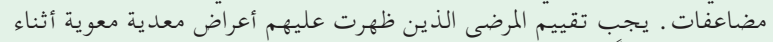

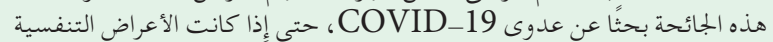
غائبة .
\end{abstract}

To describe a patient's condition and clinical progress, admitted to King Abdulaziz University Hospital, Jeddah, Kingdom of Saudi Arabia with Coronaviruses disease-19 (COVID-19) infection who presented initially with gastrointestinal symptoms. The novel COVID-19 disease does not only affect the respiratory tract but also affects other parts of the body. A 23-year old male patient came to the emergency room suffering from acute abdominal pain and vomiting. The patient appeared to have a complicated course of infection with gastrointestinal manifestations and a perforated duodenal ulcer without any respiratory symptoms. Laboratory and radiographic investigations revealed positive results for COVID-19. The chest CT showed a ground-glass appearance. Accordingly, the patient was referred for an emergency laparotomy and was treated mainly for a perforated duodenal ulcer. Unfortunately, the patient died due to several complications. Patients presenting with gastrointestinal symptoms during this pandemic should be assessed for COVID-19 infection, even if respiratory symptoms are absent.

Keywords: duodenal ulcer, coronavirus, GI bleeding, case report, COVID-19

Saudi Med J 2021; Vol. 42 (9): 1036-1040 doi: 10.15537/smj.2021.42.9.20210224
From the Department of Emergency medicine, College of medicine, King Abdulaziz University, Jeddah, Kingdom of Saudi Arabia.

Received 21st March 2021. Accepted 18th June 2021.

Address correspondence and reprint request to: Dr. Ghada A. Merdad, Department of Emergency medicine, College of medicine, King Abdulaziz University, Jeddah, Kingdom of Saudi Arabia. E-mail: ghada.merdad@gmail.com

ORCID ID: https://orcid.org/0000-0002-6043-9133

Coronaviruses disease (COVID) consist of large, enveloped, single-stranded ribonucleic acid (RNA) with crown-like spikes on their surfaces. They represent a huge family of viruses with different symptoms that might range from typical common cold to serious respiratory symptoms. ${ }^{1,2}$ Coronavirus spikes are proteins that bind to a receptor in host cells before interacting with it. In human cells, they exhibit a high binding affinity for angiotensin-converting enzyme-2 (ACE2). ${ }^{2}$ Coronaviruses have 4 main subgroups: $\alpha$, $\beta, \gamma$, and $\delta$. There are 3 commonly identified human coronaviruses: severe acute respiratory syndrome coronavirus (SARS-CoV), Middle East respiratory syndrome (MERS), in addition to the newly emerged SARS-COV-2 which causes the Coronavirus disease-19 (COVID-19). ${ }^{1}$ The phylogenetic analysis of COVID-19 showed that it is a member of the beta coronaviruses linked to SARS-COV and MERS. ${ }^{2,3}$ Fever, nonproductive cough, dyspnea, myalgia, lethargy, normal or reduced leukocyte count, pneumonia, organ malfunction, and death are just a few of the symptoms that COVID-19 can cause. ${ }^{3}$ With the recurrence of gastrointestinal tract (GIT) symptoms in COVID patients, researchers surprisingly found a significant relationship between a respiratory syndrome triggered by COVID-19 and GIT manifestations. The massive secretion of ACE2 in COVID-19 patients can explain this association in the GIT. ${ }^{2,4}$ Accordingly, several GIT clinical manifestations have been reported in COVID patients, such as diarrhea, nausea, vomiting, peptic ulcers, and upper or lower GI bleeding. ${ }^{5,6}$

A peptic ulcer is a prevalent disease mainly caused by Helicobacter pylori infection or frequent administration 
of non-steroidal anti-inflammatory drugs (NSAIDs). It is often diagnosed by coffee ground emesis and usually requires further exploration through endoscopy or radiographic investigations. Peptic ulcers result in upper gastrointestinal bleeding. Recently, some studies have demonstrated a relationship between COVID-19 and peptic ulcers. $^{7}$

Here, we report a patient who was presented to the emergency room of a tertiary center in Jeddah, Kingdom of Saudi Arabia (KSA) with typical symptoms of acute abdominal pain and was unexpectedly diagnosed with COVID-19.

Case Report. A 23-year-old Indian man, who was otherwise healthy and work as a private car driver, presented to the emergency room of King Abdulaziz University Hospital on the July 13, 2020 with a history of generalized abdominal pain for 3 days.

Clinical finding. He was complaining of nausea, melena for 3 days, and subjective fever which started 2 days before presentation. He was administered paracetamol, which showed a good response. He denied any history of vomiting or heartburn; he also denied any history of cough, chest pain, or breathing difficulty. He had no history of medication intake or substance abuse and had no history of previous surgery. On examination, he had normal vital signs except for tachycardia (heart rate $=120$ ), with normal random blood sugar and temperature. He appeared pale and in agony because of pain. Physical examination of the chest was normal, with tenderness over the right side of the abdomen, which was associated with regurgitation and rigidity throughout the abdomen. Rectal examination revealed evidence of melena. Based on the signs and symptoms of the patient, a differential diagnosis was made between suspected mesenteric ischemia, complicated appendicitis, or perforated peptic ulcer.

Diagnostic testing. Blood investigations were conducted, including cross-matching of packed red blood cells. Laboratory tests were as follows: white blood cell (WBC): 21.69, lymphocyte count: $2.33 \mathrm{~K} / \mu \mathrm{L}$, neutrophil count: $17.13 / \mu \mathrm{L}$, hemoglobin: $4 \mathrm{mmHg}$, lactic acid: 0.9 , sodium $(\mathrm{Na}): 127$, potassium $(\mathrm{K}): 2.8$, urea: 14.5. Chest radiography, erect/supine abdominal radiography and abdominal computed tomography (CT) with contrast were obtained, as shown in Figures $1 \& 2$.

Disclosure. Authors have no conflict of interests, and the work was not supported or funded by any drug company.

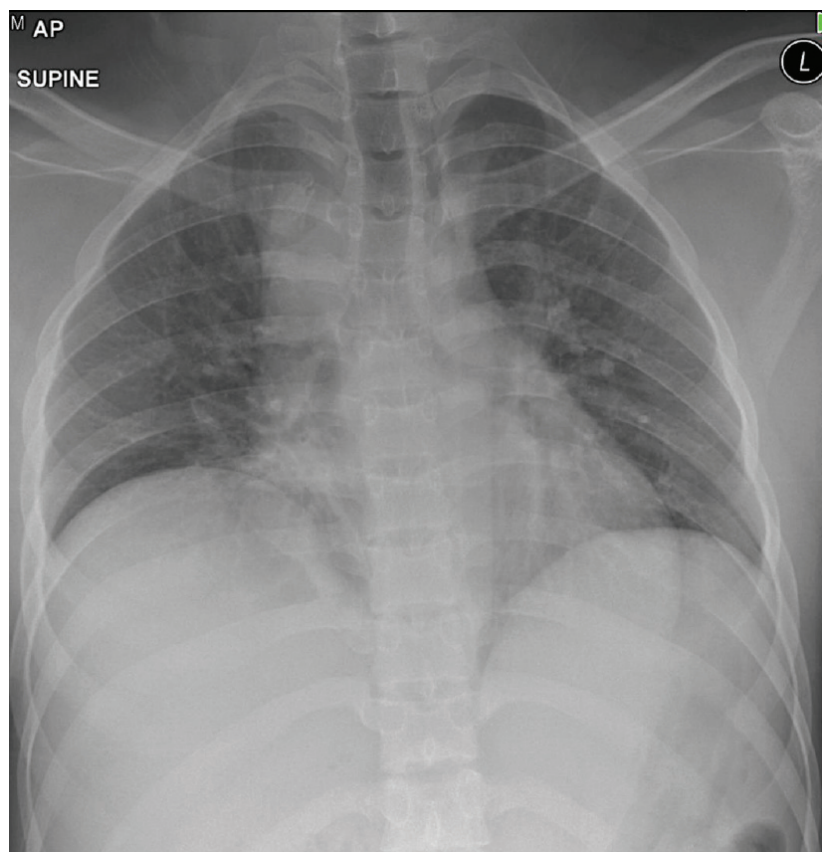

Figure 1 - Plain x-ray, anteroposterior view in supine position shows no evidence of infiltration.

Therapeutic intervention. Initial management was initiated using intravenous fluid boluses (normal saline) and intravenous omeprazole. A nasogastric tube was inserted, which showed a large amount of coffee ground emesis. Urgent contrasted CT abdomen was ordered to rule out a perforated viscus. Computed tomography showed a perforated duodenal ulcer and a ground-glass appearance on lower chest scans. On the same day, polymerase chain reaction (PCR) of nasopharyngeal swabs was investigated for SARS-CoV-2. The PCR results returned positive on the second day of COVID-19 infection testing. Ultimately, the surgical team was consulted, and the patient was referred for emergency laparotomy. He immediately went to the operation theater for primary repair of duodenal perforation with an omental patch and incorporated a jejunostomy feeding tube. Intraoperative findings included the presence of a large ulcer between the first and second parts of the duodenum with retroperitoneal leakage.

Follow-up and outcomes. The patient was then admitted to the isolation unit of the intensive care unit (ICU). Repeated PCR 14 days later tested negative for SARS-CoV-2, and the patient's condition started to show further improvement until the 16-day postoperation when his condition started to deteriorate. The patient developed persistent perforation, leakage, and hematoma collection in the abdomen. He underwent multiple laparotomies for resection and anastomosis. 


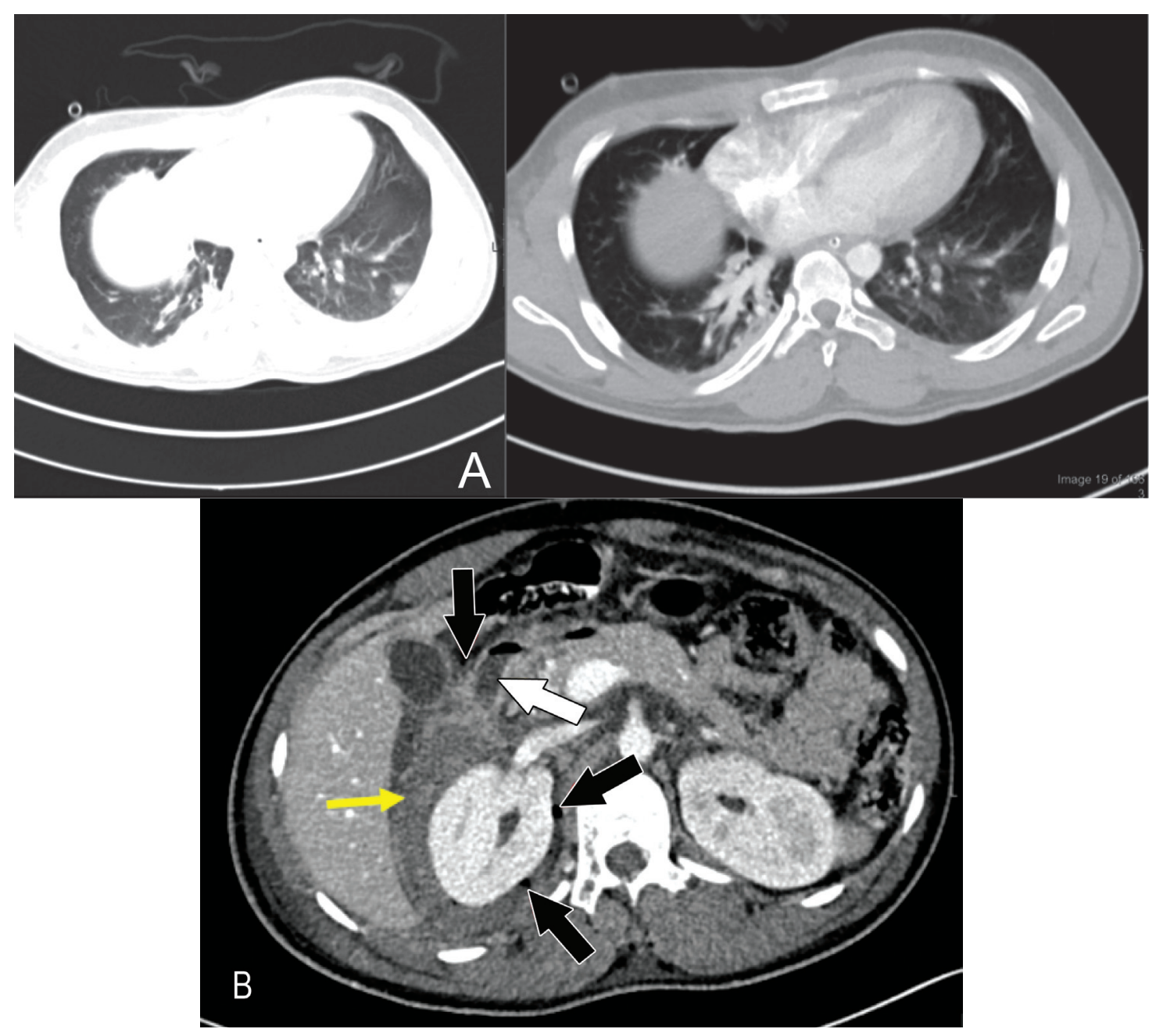

Figure 2 - High-resolution CT scan oflower chest-transverse section. Showed ground-glass opacity appearance. A) Axial cut of CT abdomen. B) Demonstrates both intraperitoneal and retroperitoneal foci of air suggesting ruptured viscous (black arrow). Yellow arrow demonstrates retroperitoneal collection containing foci of air in the paraduodenal region and extending to right perinephric and right parade along space with high density material. White arrow points at the enhancing duodenal wall which may reflect underlying inflammation.

During the hospitalization period of the patient, he developed thrombosis in the internal jugular vein (IJV), therefore, heparin infusion was initiated. Later, the patient's condition deteriorated due to evolving cardiac arrhythmias and respiratory failure. Uneventfully, the patient started to show no improvement, developed multiorgan failure, and died 2 months after admission.

Figure 3 refers to the timeline of a brief and clear description of the case.

Discussion. The COVID-19 pandemic has demonstrated significant uncertainty in the scientific research community due to the novel consequences of the virus. Accordingly, many studies have been conducted to understand the transmission mechanism, pathogenesis, and clinical manifestations of the virus.
Additionally, clinicians became more aware of the infection's extra-respiratory manifestation, which is currently under investigation.

Although the COVID-19 infection mainly presents with respiratory manifestations, some gastrointestinal tract symptoms have been reported, such as diarrhea, nausea, vomiting, and abdominal pain. Currently, these gastrointestinal manifestations are increasingly observed among COVID-19 patients. $^{5}$ In the present paper, we describe the clinical course of a patient who presented to our hospital in Jeddah, KSA, with classic complaints suggestive of complicated peptic ulcer disease without any respiratory symptoms. After laboratory and radiographic investigations, the patient was diagnosed with COVID-19 and had a complex yet successful 
A 23-year old Indian man otherwise healthy and works as a private driver, presented to emergency department with abdominal pain for 3 days

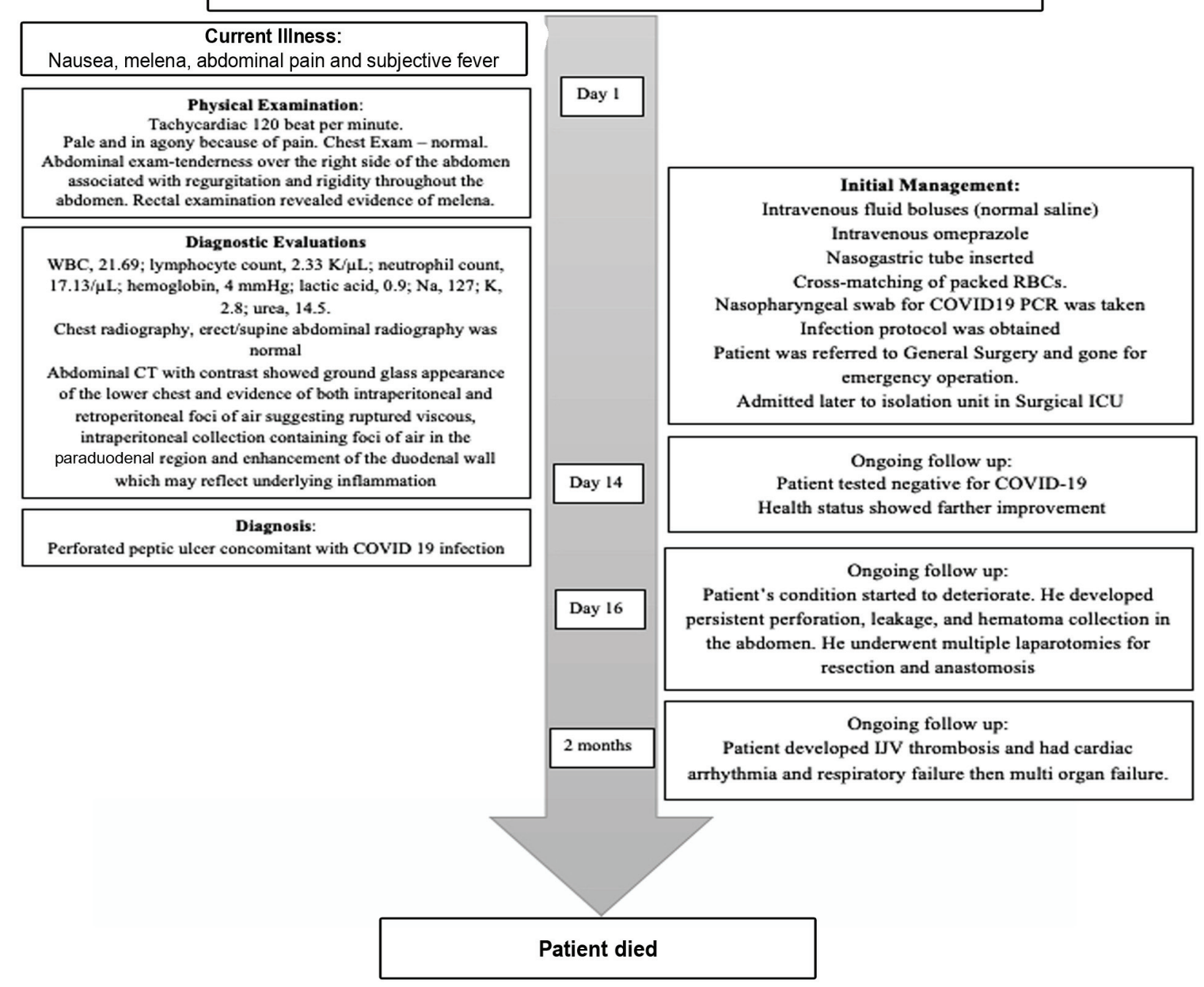

Figure 3 - The timeline figure for brief and clear description of the case.

treatment course. Globally, recent studies have revealed a significant correlation between COVID-19 and peptic ulcer disease. In the United States, Safari et $\mathrm{al}^{8}{ }^{8}$ demonstrated that COVID-19 uses angiotensinconverting enzyme 2 (ACE2) as an entry receptor to interact with cells of the host. Hence, tissues that have high ACE2 expression, such as lungs and adipose tissues of the visceral area, could be a potential target for this new virus. Safari et al, ${ }^{8}$ reported that adipose tissue expresses more ACE2 than the lungs. Accordingly, this finding can explain the high incidence of gastrointestinal symptoms among COVID-19 patients.

In Northern Italy, Mauro et al, ${ }^{5}$ described the endoscopic findings of COVID-19 patients who were presented with gastrointestinal manifestations. Mauro et $\mathrm{al},{ }^{5}$ reported that active ulcers were identified in almost half of the patients (44\%), while erosive hemorrhagic gastritis was the second most frequent endoscopic finding in $22 \%$ of patients.

Another case series in Italy by Melazzini et al, ${ }^{9}$ reported 5 patients presenting with gastrointestinal symptoms without any respiratory symptoms. All of them had a positive PCR for COVID-19. Melazzini et al, ${ }^{9}$ showed that the 5 patients were diagnosed with a peptic ulcer on admission without any previous history of peptic ulcer or Helicobacter pylori infection. However, it should be noted that all patients had comorbid conditions. Melazzini et al, ${ }^{9}$ showed that gastric and 
duodenal ulcers were the most common diagnoses, while 2 patients had more than one ulcer. Furthermore, Melazzini et al, ${ }^{9}$ showed that one patient experienced rebleeding after 2 treatment days.

In the present case report, the patient was admitted to our hospital in Jeddah, KSA, with significant gastrointestinal manifestations suggestive of perforated peptic ulcers, requiring a referral to the surgical team for an emergency laparoscopy. Subsequently, the patient developed several complications with persistent perforation and leakage and required multiple operations for correction. It should be mentioned that the patient did not suffer from any respiratory symptoms either on admission or during hospitalization.

Furthermore, He et $\mathrm{al}^{10}{ }^{10}$ reported the case of a COVID-19 patient with a history of travel to Wuhan (the first point COVID-19) who developed a duodenal bulb ulcer after weeks of infection. He et al, ${ }^{10}$ showed that the patient had a successful emergency operation, which was the first of its kind in Hunan province.

Another patient reported in the United States by Kangas-Dick et $\mathrm{al}^{4}{ }^{4}$ was admitted with typical coronavirus symptoms and was accidentally diagnosed with upper GIT perforation. Also De Nardi et $\mathrm{al}^{6}{ }^{6}$ reported an uncommon COVID-19 patient who presented with abdominal pain and distention. De Nardi et al, ${ }^{6}$ revealed that x-ray and CT scans showed ascending colon perforation. The patient had a complicated clinical course during hospitalization.

The present case reports is the first patient in our hospital with abdominal symptoms without any respiratory manifestations. Accordingly, the diagnosis of COVID-19 should be considered for all patients who suffer from acute abdominal symptoms, even in the absence of any respiratory symptoms. However, future studies with a more robust design are required to confirm the present report's findings and recommendations on a larger scale.

In conclusion, patients with COVID-19 are increasingly presenting with extra-respiratory manifestations. These patients are at a higher risk of complications and deterioration due to misdiagnosis of COVID-19 at the early stages of the infection. Gastrointestinal manifestations are among the most frequent symptoms reported in COVID-19 patients. Hence, patients who complain from acute abdominal symptoms during the COVID-19 pandemic should be conventionally screened for COVID-19 by PCR. Future large and prospective studies on the clinical course of extra-respiratory manifestations of COVID-19 are highly recommended.

Acknowledgment. The authors gratefully acknowledge Editage (www.editage.com) for English language editing.

\section{References}

1. Jaiswal NK, Saxena SK. Classical Coronaviruses. Singapore: Springer; 2020. p. 141-150.

2. Xu X, Chen P, Wang J, Feng J, Zhou H, Li X, et al. Evolution of the novel coronavirus from the ongoing Wuhan outbreak and modeling of its spike protein for risk of human transmission. Sci China Life Sci 2020; 63: 457-460.

3. Wang D, Hu B, Hu C, Zhu F, Liu X, Zhang J, et al. Clinical characteristics of 138 hospitalized patients with 2019 novel coronavirus-infected pneumonia in Wuhan, China. JAMA 2020; 323: 1061-1069.

4. Kangas-Dick A, Prien C, Rojas K, Pu Q, Hamshow M, Wan E, et al. Gastrointestinal perforation in a critically ill patient with COVID-19 pneumonia. SAGE Open Med Case Rep 2020; 8: 2050313 X20940570.

5. Mauro A, De Grazia F, Lenti MV, Penagini R, Frego R, Ardizzone $S$, et al. Upper gastrointestinal bleeding in COVID-19 inpatients: incidence and management in a multicenter experience from Northern Italy. Clin Res Hepatol Gastroenterol 2021; 45: 101521.

6. De Nardi P, Parolini DC, Ripa M, Racca S, Rosati R. Bowel perforation in a Covid-19 patient: case report. Int J Colorectal Dis 2020; 35: 1797-1800.

7. Mao R, Qiu Y, He JS, Tan JY, Li XH, Liang J, et al. Manifestations and prognosis of gastrointestinal and liver involvement in patients with COVID-19: a systematic review and meta-analysis. Lancet Gastroenterol Hepatol 2020; 5: 667-678.

8. Safari S, Keyvani H, Malekpour Alamdari N, Dehghanian A, Razavi Hashemi M, Nemati Honar B, et al. Abdominal surgery in patients with COVID-19: detection of SARS-CoV-2 in abdominal and adipose tissues. Ann Surg 2020; 272: e253-e256.

9. Melazzini F, Lenti MV, Mauro A, De Grazia F, Di Sabatino A. Peptic ulcer disease as a common cause of bleeding in patients with coronavirus disease 2019. Am J Gastroenterol 2020; 115 : 1139-1140.

10. He L, Zhao W, Zhou W, Pang P, Liao Y, Liu J. An emergency surgery in severe case infected by COVID-19 with perforated duodenal bulb ulcer. Ann Surg 2020; 272: e35-e37. 